\title{
ASSESSMENT OF THE EVOLUTION IN QUALITY FOR XAMARIN ANDROID MULTIMEDIA APPLICATIONS
}

\author{
Cosmin TOMOZEI \\ Vasile Alecsandri University of Bacău, Romania \\ cosmin.tomozei@ub.ro
}

\begin{abstract}
The large-scale adoption of mobile and smart devices, as well as the orientation of business and services to the mobile paradigm, inferred that study of evolution in quality of software became progressively much more oriented to the evaluation of mobile applications. Consequently, the process of software development and especially the software testing field have been subjected to major transformations led by the process of software reengineering. Nowadays, the industry has known new processes, product lines and projects but the most important fact is that new principles have been defined. The shifting of paradigm from the desktop and web to mobile applications created the premises for different approaches on user interaction user experience through smaller size screens, touch gestures and new scenarios. This paper analyses the evolution of XAMARIN mobile platform for.NET in terms of quality for the development of image and sound applications by the way in which the integration of multimedia has transformed the process of applications development.
\end{abstract}

Keywords: software evolution, mobile applications, multimedia, reengineering.

JEL classification: L86

DOI: $10.24818 / \mathrm{ie} 2020.01 .07$

\section{Introduction}

The world-scale adoption of mobile technologies is obvious in all the economic sectors, from manufacturing and logistics to online shopping, online banking and eHealth [1]. This process created the premises for many technological innovations in software development. The transition from classical software applications such as desktop and web to mobile engaged the need of rebuilding almost every aspect related user interaction and user experience. These transformations have mainly been made because of the different user gestures and of the different input devices. The use of touch sensitive screens of various sizes and numerous resolutions recommended a distinctive way of designing the user interfaces, by re-thinking sizes, gestures and user scenarios.

When building Android applications, either on Android Studio with Java language or on Visual Studio and Xamarin, developers should be focused on creating user interfaces as activities and each activity should behave according to their well-defined life cycles. As one application may include many activities, it is necessary that a high level of concern regarding the data should be directed to saving and restoring of the user data between states. The development of multimedia applications requires that specialized classes, such as Android.Provider.MediaStore should be implemented in order to store the media files, as a result of actions described by Android Intents.

Every mobile application should comply to the standards described in [2], especially to the usability standard that should lead the development teams to the evolution of quality in user interaction. Consequently, each version of the app should bring a leap forward in usability and to meet the expectations of the user in a very simple manner. Additional quality characteristics 
also mentioned in [2], by referring to functionality, reliability and portability. Responsiveness in this case plays a decisive role, one application interface being designed as based on density independent pixels in order to the application UI to be scaled for multiple devices with different screen sizes and resolutions.

\section{Evolution of multimedia applications on Xamarin Platform}

The integration of multimedia on mobile applications has always been a valuable technique for attracting users and for offering an enjoyable user experience. Sound, image and animation provided a sense of completeness and joy to the users. However, multimedia on mobile always required both hardware and software resources, materialized in design and development costs. Capturing and playing image and sound have always been concerning mobile application developers. Since the beginning of Android SDKs, intents have been used in order to achieve these requests. An intent represents a message which is sent to another component with the aim of taking a specific action [3]. Intents are utilized on the purpose to start a specific activity, starting a specific service or for broadcasting a message. Working with media also requires that the necessary permission are offered to the application. The apps that capture of images and sound should have the corresponding permissions in the applications manifests file, in which the following elements should be included:

<uses-permission android:name="android.permission.CAMERA" />

<uses-permission android:name="android.permission.WRITE_EXTERNAL_STORAGE" />

These permissions ensure that the event of capturing the image from camera, materialized by an intent is allowed and the resulting image has the facility of being saved on the external memory. The image file has further the possibility of being synchronized on Cloud [4]. Going forward by working with video and sound needs having a higher quality of service (QOS) so as to provide an accurate and uninterrupted user experience.

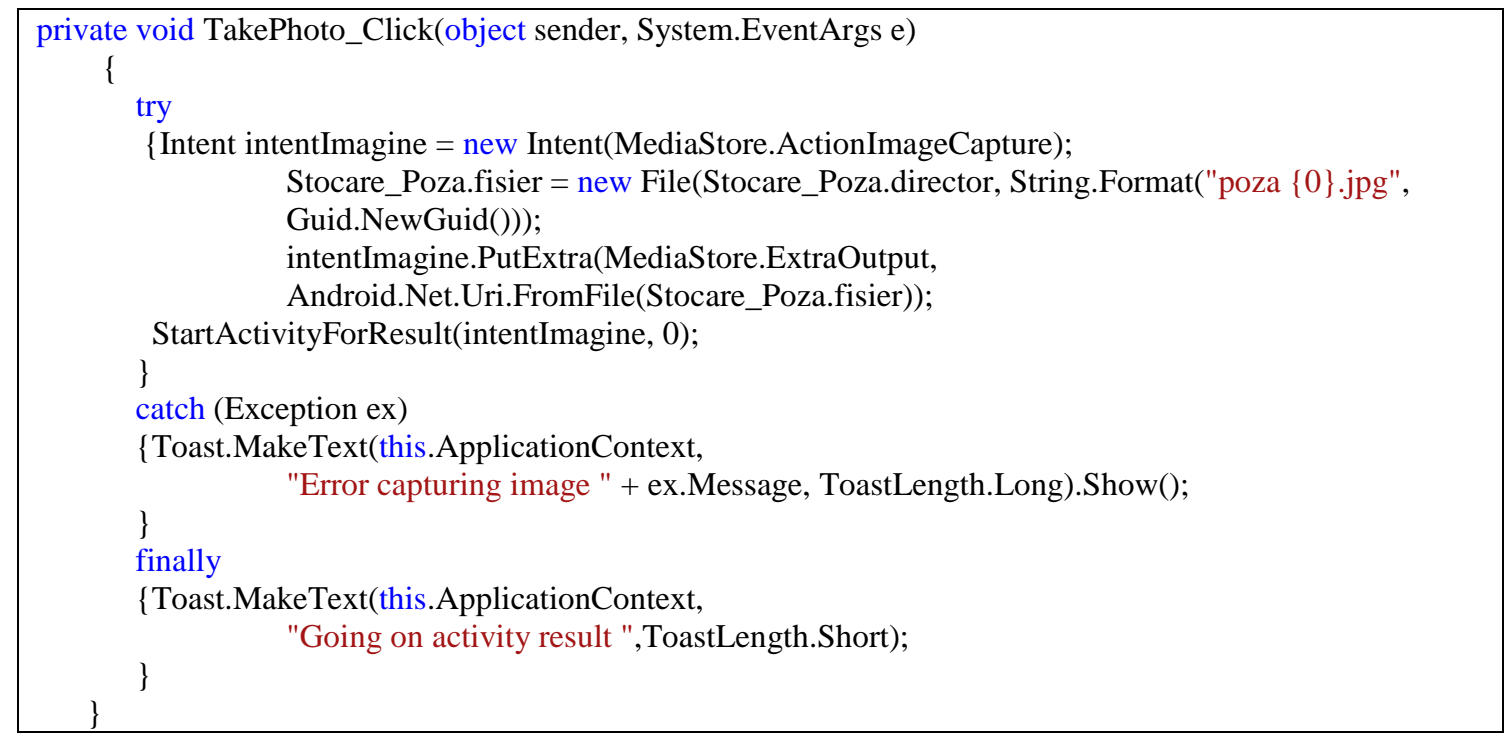

Figure 1. Android Xamarin C\# take photo method

Figure 1 presents a method of taking a photo based on an ActionImageCapture intent. This intent is sent to the device camera. The camera further takes an picture and sends it to the device. Should any exceptions be generated, a message of error will be sent as a toast message to the user. In development mode on the stage of functional testing [5] it is advisable that the exception message - ex.Message should be shown during tests. In production, this type of 
technical messages should be hidden in order not to affect the degree of usability. The tests should take into consideration the in-app permissions, the device permissions and the behavior of the application regarding the access on the external memory.

Furthermore, if applications need the streaming of multimedia such as game playing [6] or video and sound [7] downloading from Cloud, a certain level of quality and connectivity should be continuously provided. Before any sync on libraries such as Google Photos or OneDrive, the captured images should be managed by the MediaStore Class. In Xamarin, the MediaStore class is derived from the Java language Object class, as public sealed class MediaStore : Java.Lang.Object. Each media element is stored through MediaStore in specific tables, such as the MediaStore.Images table, the MediaStore.Video table or the MediaStore.Audio table. It is also compulsory to give the necessary permissions for reading and writing on external files. When syncing or consuming multimedia, mobile users experience is greatly affected by intermittent connectivity, which should be managed by a model for users' connection along their path.

\section{Mobile multimedia applications reengineering quantitative indicators}

Due to the numerous versions of Android devices and of the high frequency of code transformation within the platform SDKs, frequent updates and adaptive maintenance procedures are needed for the existing applications. Both C\# and Java applications need both manual and automatic testing suites [8]. Consequently, re-writing parts of software generate intrinsic redundancy mainly found as duplicated sequences or duplicated modules of code. In order to have a clear image on the code duplication, at first the code duplication indicator is defined.

The code duplication indicator, IDC, is given by the relation (1):

where:

$$
I D C=\frac{N r D u p}{N r \operatorname{Comp}}
$$

NrDup - the number of duplicated components, as modules or methods;

$\mathrm{NrComp} \mathrm{-} \mathrm{the} \mathrm{total} \mathrm{number} \mathrm{of} \mathrm{components} \mathrm{in} \mathrm{the} \mathrm{applications.}$

This indicator identifies the existing redundant components for the structure which are further either elliminated or rewritten for other functionalities [9]. Two modules might look or might not look the same but behaves the same. If one module becomes deprecated, it should be eliminated because of the lack of functionality or because of the lack of further support. A particular case is the implementation of the Camera and Camera2 APIs, discussed in part four of this paper.

In case any decision of the elimination of components is made, the indicator of component elimination should be also defined.

The indicator of component elimination, IDEL, is described in the relation (2)

where:

$$
I D E L=\frac{N r D e l}{N r C o m p}
$$

$\mathrm{NrDel} \quad-$ the number of components eliminated from the application structure;

NrComp - the number of components of the mobile application.

The indicator of component elimination represents the weight of the elements identified as being useless for any further transformation of the existing mobile application.

One way to reflect the code transformation through the process of reengineering is the code refinement indicator.

The code refinement indicator, $I R A F$, is shown in (3) by the following relation: 
www.conferenceie.ase.ro

$$
I R A F=1-\frac{N r D u p}{N r \operatorname{Comp}}=\frac{N r \operatorname{Comp}}{N r \operatorname{Comp}}-\frac{N r D u p}{N r \operatorname{Comp}}
$$

The relation (3) is equivalent with (4):

$$
I R A F=\frac{N r C o m p-N r D u p}{N r C o m p}
$$

The indicator of code reuse, IREUS, is defined in (5) as the difference between the indicator of code refinement and the indicator of component elimination:

$$
I R E U S=I R A F-I D E L
$$

These indicators should be based on pre-existing test case scenarios, for each of the mobile application functionality. Every information concerning the existing classes of the application proves to be important for obtaining suitable tests. A reliable technology for transformation of comments in test oracles is studied in [8]. It clearly shows that comments are especially important for documenting the methods of the existing classes. The process of reengineering is further conducted more efficiently if the software components are documented in an appropriate manner.

The higher level of reusability is achieved, the more productive reengineering becomes. Reusability of software libraries reduces both the costs and the duration of the development cycle.

\section{Reengineering Camera functionalities in mobile applications}

One relevant side of the evolution of mobile multimedia applications on Android consists of the transition from the Camera class to the Camera2 class, which according to [13] is much more complex but also very complicated. Developers should answer to the question whether it is reliable to re-engineer the existing software libraries or to re-write the code from scratch.

The image capture action has been implemented since the API level 3, meaning that the first release of the image capturing method was on Android version 1.5 Cupcake.

[Register("ACTION_IMAGE_CAPTURE", ApiSince = 3)]

public const string ActionImageCapture = "android.media.action.IMAGE_CAPTURE";

Working with Camera [10] became deprecated (obsolete) and nowadays it is recommended that instead of Camera, applications should be developed with Camera2. The class became deprecated on API level 21, namely Android 5.0 Lolllipop. One specific aspect of the Camera class is that it is not thread-safe [11] and the class methods should not be called from multiple threads in parallel.

Since API level 21, the Camera2 API namespace [12] with the CameraCaptureSession class have been applied. Camera2 API has been mainly utilized in multimedia applications development because it has more characteristics and works more efficiently for image capturing and further processing. Camera2 replaces the deprecated class and provides more functionalities regarding the way in which multiple requests are received at once. It then processes all of the requests in the order they arrived.

[Register("android/hardware/camera2/CameraCaptureSession", DoNotGenerateAcw = true, ApiSince = 21)] public abstract int Capture(CaptureRequest request, CaptureCallback listener, Handler handler); 
The method "Capture" is defined as abstract which means that the method has to be overridden by the derived classes. In order to capture images, the application has to create a capture session at first.

In table 1, a contrastive analysis is made based on the characteristics, namely attributes and methods of Camera and Camera2 classes.

Table 1. Comparison between Camera and Camera2 classes

\begin{tabular}{|l|l|l|}
\hline Characteristics & Camera & Camera2 \\
\hline Deprecated & Yes & No \\
\hline Default settings & getParameters() & getCameraCharacteristics(String) \\
\hline Thread safe & No & Yes \\
\hline Image Preview & startPreview() $\begin{array}{l}\text { Create a capture session to start } \\
\text { the preview } \\
\text { Use a surface texture } \\
\text { CameraCaptureSession.StateC } \\
\text { allback() }\end{array}$ \\
\hline Take photo & $\begin{array}{l}\text { Intent(MediaStore.Actio } \\
\text { nImageCapture) }\end{array}$ & $\begin{array}{l}\text { CameraCaptureSession.CaptureCallba } \\
\text { ck }\end{array}$ \\
\hline $\begin{array}{l}\text { In order processing } \\
\text { request the camera the the picture } \\
\text { should be released } \\
\text { explicitly. }\end{array}$ & $\begin{array}{l}\text { Multiple requests ordered, threaded, } \\
\text { pipelined. }\end{array}$ \\
\hline
\end{tabular}

Reengineering multimedia mobile applications entails that the methods implemented for camera images processing to be rewritten as to implement the abstract classes of Camera2 API. Developers should mainly be focused on understanding the main differences between the deprecated class and the actual class. At first, a texture view component is necessary and a surface texture view listener for initiating the component. Secondly, a camera device state callback is fired, so as to get the status of the actual hardware camera. Afterwards, the camera capture session callback is executed in order to take the picture and then the picture is sent to be written on the external memory.

\section{Conclusions}

This study introduced a method of analysis concerning mobile multimedia applications development. On the one hand, the large-scale adoption of mobile technologies inferred that developers got re-oriented from classical desktop and web applications to mobile. On the other hand, mobile applications have to evolve fast, as APIs and SDK evolve, in order not to become deprecated, unsupported or obsolete. Code transformation and adaptive maintenance often generate redundancy and code duplication. This means that at a certain stage, applications should be radically transformed through reengineering so that they re-gain clarity and quality. The evolution from the Camera to the Camera2 classes shown clearly that it is not a very simple process and it requires a certain level of effort to re-code the mobile multimedia applications. The process of reengineering of mobile applications relies on software testing and on the evolution of API SDKs. These dynamics also oblige developers to abandon some classes and APIs which became deprecated along the evolution of the operating systems versions. 
www.conferenceie.ase.ro

\section{References}

[1] S. Panichella , F. Palomba, D. Lo and Meiyappan Nagappan, "Guest Editorial: Special Issue on Software Engineering for Mobile Applications", Empirical Software Engineering, vol. 24, pp. 3249-3254, December 2019.

[2] K. Moumane, A. Idri and A. Abran, "Usability evaluation of mobile applications using ISO 9241 and ISO 25062 standards”, Springerplus, vol. 4, no. 548, April 2016.

[3] Intents and intent filters, Internet: https://developer.android.com/guide/components/intentsfilters [03,2020]

[4] Y. Kirsal, - G. Mapp and F. Sardis "Using Advanced Handover and Localization Techniques for Maintaining Quality-of-Service of Mobile Users in Heterogeneous Cloud-Based Environment", Journal of Network and Systems Management, vol. 27, pp. 972-997, March 2019.

[5] P. Tramontana, D. Amalfitano, N. Amatucci and·A. R. Fasolino, "Automated functional testing of mobile applications: a systematic mapping study", Software Quality Journal, vol. 27, pp. 149-201, March 2019.

[6] L. Valente, B. Feijo, J. C. S. do Prado Leite, "Mapping quality requirements for pervasive mobile games", Requirements Engineering, vol. 22, pp. 137-165, September 2015.

[7] I. Himawan, W. Song and· D. Tjondronegoro, "Impact of automatic region-of-interest coding on perceived quality in mobile video", Multimedia Tools and Applications, vol. 76, pp. 785-813, 2017.

[8] A. Goffi, A. Gorla, M. D. Ernst and M. Pezzè, "Automatic Generation of Oracles

for Exceptional Behaviors" Proceedings of the 25th International Symposium on Software Testing and Analysis, ISSTA 2016, pp. 213-224.

[9] L. Mariani, M. Pezzè and D. Zuddas, "Augusto: Exploiting Popular Functionalities for the Generation of Semantic GUI Tests with Oracles", Proceedings of IEEE/ACM $40^{\text {th }}$ International Conference on Software Engineering, ICSE 2018, pp. 54-65.

[10] Microsoft Docs, Camera Class API, Internet https://docs.microsoft.com/enus/dotnet/api/android.hardware.camera?view=xamarin-android-sdk-9 [03, 2020]

[11] V. Terragni and M. Pezzè, "Effectiveness and challenges in generating concurrent tests for thread-safe classes" Proceedings of the 33rd ACM/IEEE International Conference on Automated Software Engineering, ASE 2018, pp. 64-75.

[12] Microsoft Docs, Camera2 Class API, Internet: https://docs.microsoft.com/enus/dotnet/api/android.hardware.camera2?view=xamarin-android-sdk-9 [03, 2020]

[13] X. Ling, "Android Camera Preview App: Camera vs. Camera2", Internet: https://www.codepool.biz/android-camera-preview-app-camera2.html [03, 2020] 